\title{
Anaplastic transformation of low-grade gliomas (WHO II) on magnetic resonance imaging
}

\author{
Barbara Bobek-Billewicz ${ }^{1}$, Gabriela Stasik-Pres ${ }^{1}$, Anna Hebda ${ }^{1}$, Krzysztof Majchrzak ${ }^{2}$, Wojciech Kaspera ${ }^{2}$, \\ Marek Jurkowski ${ }^{3}$ \\ ${ }^{1}$ Radiodiagnostic Department, Maria Sklodowska-Curie Memorial Cancer Center and Institute of Oncology, Gliwice Branch, \\ ${ }^{2}$ Department of Neurosurgery, Medical University of Silesia, Sosnowiec, ${ }^{3}$ Nuclear Medicine and Endocrine Oncology \\ Department, Maria Sklodowska-Curie Memorial Cancer Center and Institute of Oncology, Gliwice Branch, Poland
}

\begin{abstract}
Introduction: Malignant transformation among gliomas WHO II ranges between 35\% and 89\%. However, according to some reports, all gliomas WHO II undergo such transformation over time. The aim of the study was to analyse MRI parameters indicating anaplastic transformation of gliomas WHO II.

Material and methods: Forty-six consecutive patients were enrolled in the study (20 females and 26 males; range of age $36 \pm 9$ years) with supratentorial glioma WHO II. Multiparametric MR examination included morphological imaging, perfusion-weighted imaging, diffusion-weighted imaging and proton magnetic resonance spectroscopy. Group division depended on the course of disease (ST - stable group, AT - anaplastic transformation group).

Results: Subtotal tumour resection was achieved in the whole AT group, whereas in the ST group, total tumour resection was achieved in 10/29 (34\%) patients. The size of the residual tumour after surgery was statistically significantly higher in the AT group compared to the ST group (AT: $51.5 \mathrm{~cm}^{3} \pm 37.7 \mathrm{VS}$. ST: $29.0 \mathrm{~cm}^{3} \pm 37.9 ; p=0.011$ ). Contrast enhancement in the AT group occurred in 5/11 (45\%) of tumours and in none of the patients' areas of contrast enhancement were resected during surgery/biopsy. However, the initial MR showed contrast enhancement in 10/29 (34\%) of patients in the ST group. The areas of contrast enhancement were totally resected in all patients. Compared to the ST group tumours that underwent anaplastic transformation had statistically significantly higher values of mean $n r C B V$ and max $n r C B V$ on the initial $M R$, the follow-up and final MR examinations. However, statistically significant differences between the groups in $A D C$ values were observed on the follow-up and final MR whereas mean Cho/Cr and mean Cho/NAA were observed as late as on the final MR examination.

Conclusions: Multiparametric MR examination allows the detection of LGGs with high probability of rapid anaplastic transformation and the detection of transformation prior to the occurrence of contrast enhancement. The value of $n r C B V$ is the most useful in the diagnosis of anaplastic transformation. The resection of contrast enhancement area of the tumour significantly increases time to anaplastic transformation of LGGs.
\end{abstract}

Key words: anaplastic transformation, low-grade glioma, MR imaging. 


\section{Introduction}

According to the National Cancer Registry, malignant tumours comprised about 156000 new cases and 93000 deaths in Poland in 2009. Primary brain tumours were the $11^{\text {th }}$ most common cause of malignant tumour in males $(2.1 \% ; 1444$ new cases in 2009 ) and the $15^{\text {th }}$ most common in females $(2.0 \%$; 1362 new cases in 2009). Brain tumour mortality rate has remained stable since 1965 and according to the latest report, it was $2.8 \%(7.98 / 100000)$ in males and $3.6 \%(7.48 / 100$ 000) in females [16]. Similar percentages of cases and deaths are reported in Europe and in the USA [11,26,35].

Gliomas comprising more than $40 \%$ of intracranial tumours constitute high-grade gliomas (HGGs) in $70-80 \%$ of cases [30]. Surgical excision with subsequent radio- and chemo-radiotherapy is the gold standard in treatment of HGGs. However, low-grade gliomas (LGGs) are mainly treated surgically and in some cases complimentary radiotherapy is administered. According to the 2011 diagnostic-therapeutic recommendations by the Polish Society of Clinical Oncology (PTOK), postoperative radiotherapy may be abandoned in the case of total surgical resection of oligodendroglioma, gemistocytic astrocytoma, if the tumour did not show contrast enhancement on preoperative (CT and MR) examination and if the patient was < 40 years of age. Postoperative chemotherapy may be considered in oligodendroglioma with $1 \mathrm{p} / 19 \mathrm{q}$ loss of heterozygosity $[3,21,36]$. Gliomas WHO II which predominantly affect patients at the age of 30-40 are mostly characterised by diffuse infiltration of multiple cerebral structures [30]. Malignant transformation to a higher grade (WHO III and IV) in this group of tumours ranges between $35 \%$ and $89 \%$. However, according to some reports, all gliomas WHO II undergo such transformation over time $[37,48]$. The median survival in patients with glioma WHO IV is $<2$ years, whereas WHO II is 5-10 years. It is believed that the early detection of factors/parameters indicating anaplastic transformation may improve the prognosis $[21,30,40]$.

Imaging studies are currently the standard method for early detection of changes in biology and malignancy of the tumour and precede the surgical procedure or stereotactic biopsy which is associated with an increased complication risk $[21,27]$. Malignancy is characterised by significant vascular proliferation within the tumour (neoangiogenesis)
$[6,22,30,39,47]$. According to the "angiogenic switch" theory, LGG progression to HGG must be accompanied by vascular proliferation within the tumour $[22,47]$. Neoangiogenesis results in increased relative cerebral blood flow (rCBF) and relative cerebral blood volume (rCBV) and at a later stage contrast tumour enhancement on MR examination. Contrast enhancement as a marker of anaplastic transformation presents some disadvantages and poses some limitations. It appears late and its appearance proves that anaplastic transformation has already occurred. Additionally, it is a non-specific feature, resulting from the blood-brain barrier (BBB) breakdown due to e.g. inflammatory and ischaemic changes, radiotherapy as well as lack of the normal BBB in vasculature formed by neoangiogenesis. The extent of contrast enhancement also depends on the dose of glucocorticoids and even on the examination technique $[5,17,35]$. Although contrast enhancement is considered to be typical of HGGs, it should be remembered that about $40 \%$ of high-grade tumours do not show contrast enhancement whereas $10-39 \%$ of LGGs show contrast enhancement $[21,28,30,35,37,45]$.

The aim of the study was to analyse MRI parameters indicating anaplastic transformation of gliomas WHO II.

\section{Material and methods Material}

Forty-six consecutive patients were enrolled in the study (20 females and 26 males; range of age 36 \pm 9 years) with supratentorial glioma WHO II. Patients were diagnosed in 2005-2011 at the Radiodiagnostic Department, Cancer Centre and Institute of Oncology in Gliwice, Poland. Surgical procedure/biopsy was performed at the Department of Neurosurgery, Medical University of Silesia, Sosnowiec, Poland. Multiparametric MR examination included morphological imaging, perfusion-weighted imaging (PWI), diffusion-weighted imaging (DWI) and proton magnetic resonance spectroscopy ( ${ }^{1} \mathrm{H}$-MRS). Pathologic diagnosis was confirmed either by a surgical procedure or biopsy. In the majority of patients, i.e. (36/46) $78.3 \%$, surgery was followed by excised area radiotherapy and/or radiotherapy for residual tumour 2 to 9 months after the diagnosis. The follow-up between the pathological diagnosis and the final follow-up MR examination was at least 18 months except for patients with earlier anaplastic transformation. 


\section{Methods}

Magnetic resonance examinations were performed on a 1.5T (Magnetom Avanto, Siemens) or a 3.0T scanner (Achieva, Philips) with the standard head coil.

\section{Conventional MR imaging}

Conventional MR imaging consisted of morphological sequences of T1-weighted images before and after intravenous contrast agent application, T2-weighted images and fluid attenuated inversion recovery (FLAIR) images. Tumour volume was calculated based on T2-weighted images and FLAIR images, approximating to an ellipsoid.

\section{Perfusion-weighted imaging}

Perfusion-weighted imaging (PWI) was performed using dynamic susceptibility contrast perfusion magnetic resonance imaging (DSC MRI).

Scanner 1.5T: EPI SE (Echo Planar Imaging Spin Echo), TR/TE 1560/30 ms, Thk/gap 5.0/1.5 mm, matrix $128 \times 128$, FOV $250 \times 250 \mathrm{~mm}, 50$ data sets were acquired with a time resolution 1 per data set. TA was $1 \mathrm{~min} 26 \mathrm{~s}$.

Scanner 3.0T: EPI GE (Echo Planar Imaging Gradient Echo), TR/TE $16\left(7^{\circ}\right) / 24\left(7^{\circ}\right)$, Thk/gap 4.0/0.0 mm, matrix $64 \times 128$, FOV $230 \times 230 \mathrm{~mm}$. 50 data sets were acquired with a time resolution 1 per data set. TA was 1 min $29 \mathrm{~s}$.

A gadolinium-based contrast agent was administered at the dose of $0.1 \mathrm{mmol} / \mathrm{kg}$ with the injection rate of $6 \mathrm{~mL} / \mathrm{s}$, followed by administration of $20 \mathrm{~mL}$ bolus of saline. Parametric maps of rCBV were calculated automatically by the software provided by the scanner producer. The region of interest (ROI) which was the basis for calculating rCBV drawn manually on each layer of the parametric map on which in the correlation with morphological images the tumour was detected. ROI was drawn in the solid tumour area ignoring cortical vessels, necrotic areas and treatment-induced changes. The value of rCBV in the tumour was normalised to normal appearing white matter in the contralateral hemisphere (normalised rCBV - nrCBV). The mean nrCBV was calculated and the max nrCBV was determined in the tumour.

\section{Diffusion-weighted imaging}

Diffusion-weighted imaging (DWI) was performed using Echo Planar Imaging Spin Echo (EPI SE).
Scanner 1.5T: TR/TE 3100/99 ms, b = 0, 500, $1000 \mathrm{~mm}^{2} / \mathrm{s}$, FOV $230 \times 230 \mathrm{~mm}$; matrix $192 \times 192$, Thk/gap 5.0/1.0 mm.

Scanner 3.0T: TR/TE 3080/70 ms, b $=0$, 500, $1000 \mathrm{~mm}^{2} / \mathrm{s}$, FOV $230 \times 230$, matrix $112 \times 256$, Thk/ gap 4.0/1.0 mm.

Apparent Diffusion Coefficient (ADC) maps were calculated automatically using the software provided by the scanner producer. ROI was drawn manually on each layer of the parametric map in the solid tumour area in the correlation with morphological images. The mean ADC was calculated and the min $A D C$ was determined in the tumour.

\section{Proton MR spectroscopy}

Point resolved localised spectroscopy (PRESS) was used for single-voxel spectroscopy (SVS) and chemical shift imaging multi voxel (CSI).

Scanner 1.5T: 3D CSI - TR/TE 2000/135 ms, SVS - TR/TE 1500/135 ms.

Scanner 3.0T: 3D CSI - TR/TE 2000-1500/144288 ms, SVS - 2000-1500/144-288 ms.

Spectroscopy data were evaluated using Linear Combination of Model Spectra software (LCModel, version 6.1-4.F). Cho/Cr, Cho/NAA and NAA/Cr ratios (Choline, $\mathrm{Cr}$ - creatine, NAA - N-Acetylaspartic acid) were calculated. The voxel in SVS was placed in the solid tumour area. Spectroscopy data from every voxel placed in the solid tumour area were analysed in $3 \mathrm{D} \mathrm{CSI}$ and in the subsequent analysis, the mean Cho/Cr, Cho/NAA, NAA/Cr were used.

\section{Statistical analysis}

Continuous parameters with normal distribution were presented as mean \pm standard deviation (SD). Median and interquartile ranges were used to describe quantitative variables whose distribution differed from the standard. Significance of mean differences was tested with a $t$-Student test and an ANOVA proceeded by evaluation of normality and Levine's test. When significant effects were found, post hoc testing (NIR Fisher tests) of all possible comparisons was applied. Friedman's repeated measures ANOVA was used to test changes in not continuous values. Wilcoxon's test was used for post hoc analysis. To assess the differences between the variables, the Mann-Whitney $U$ test was used to compare two independent groups. Statistically significant $p$-levels were assumed as $<0.05$ (two-sided). 
Statistical calculations and analyses were performed with STATISTICA (StatSoft) software version 9.0.

\section{Results}

Patients were divided into three groups depending on the course of disease (Fig. 1).

ST - stable group - total/subtotal tumour resection with no relapse/progression in clinical observation and follow-up MR examinations.

AT - anaplastic transformation group - pathologically verified tumour transformation to a higher grade of malignancy (WHO grade III or IV). Time to anaplastic transformation varied between 2 and 38 months. Transformation occurred within 12 months of the follow-up in $6 / 11$ patients, between the $12^{\text {th }}$ and the $24^{\text {th }}$ month in $3 / 11$ patients and after 24 months in 2 patients.

$\mathrm{RP}$ - radiological progression group - radiological progression without pathologically confirmed transformation.

Radiological progression was detected based on the increase in tumour volume by over $40 \%$ (1 patient in the RP group) or the occurrence of contrast enhancement with the normalised max nrCBV $>1.75$ (11 patients in the AT group and 5 patients in the RP group) [17,23-26].

Initial pathologic diagnosis is presented in Table I.

The comparative analysis included 40 patients (29 from the ST group and 11 from the AT group). The selected parameters of PWI, DWI and ${ }^{1} \mathrm{H}-\mathrm{MRS}$ were analysed prior to surgery/biopsy (initial MR), after surgery/biopsy (follow-up MR) and in the AT group in the final MR examination (final MR) where anaplastic transformation was detected prior to the surgical procedure and in the ST group in the final MR follow-up in which the tumour was stable at least 18 months after pathological diagnosis (final MR). Mean time of the follow-up MR in the AT group was 3 (1-8) months and in the ST group was 3 (1-8) months. Mean time of the final MR in the AT group was 16 (3-38) months and in the ST group was 32 (18-66) months.

Subtotal tumour resection was achieved in the whole AT group, whereas in the ST group total tumour resection was achieved in 10/29 (34\%) patients. The size of the residual tumour after surgery was statistically significantly higher in the AT group compared to the ST group (AT: $51.5 \mathrm{~cm}^{3} \pm 37.7$ vs. ST: $\left.29.0 \mathrm{~cm}^{3} \pm 37.9 ; p=0.011\right)$.

Contrast enhancement on the initial MR was found in 15/40 (38\%) tumours. Contrast enhancement in the AT group occurred in 5/11 (45\%) tumours and in none of the patients, areas of contrast enhancement were resected during surgery/biopsy. However, the initial MR showed contrast enhancement in 10/29 (34\%) patients in the ST group. The areas of contrast enhancement were totally resected in all patients, in 5/10 (50\%) patients, total tumour resection was achieved and in 5/10 (50\%), subtotal tumour resection was achieved in the area of contrast enhancement.

In the AT group on the initial MR in the area of contrast enhancement, mean nrCBV, mean $\mathrm{Cho} / \mathrm{Cr}$ and mean Cho/NAA were significantly higher and the values of min $A D C$, mean $A D C$ and $N A A / C r$ were

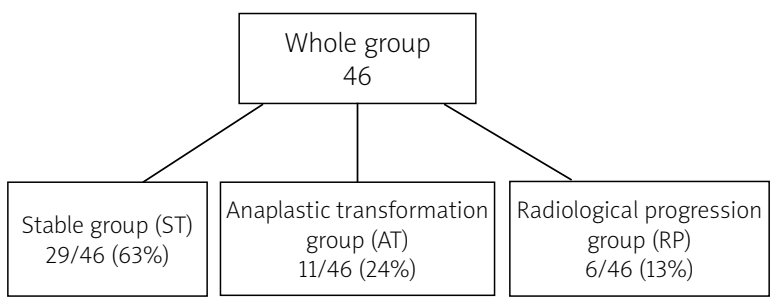

Fig. 1. Group division depending on the course of disease.

Table I. Initial pathologic diagnosis

\begin{tabular}{|lccc|}
\hline Pathologic diagnosis & ST* & AT\# & RP \\
\hline Fibrillary astrocytoma WHO II & $10 / 29(34 \%)$ & $6 / 11(55 \%)$ & $3 / 6(50 \%)$ \\
\hline Gemistocytic astrocytoma WHO II & $6 / 29(21 \%)$ & $4 / 11(36 \%)$ & $2 / 6(33 \%)$ \\
\hline Oligodendroglioma WHO II & $2 / 29(7 \%)$ & $0 / 11(0 \%)$ & $0 / 6(0 \%)$ \\
\hline Oligoastrocytoma WHO II & $11 / 29(38 \%)$ & $1 / 11(9 \%)$ & $1 / 6(17 \%)$ \\
\hline
\end{tabular}

$S T$ - stable group, AT - anaplastic transformation group, $R P$ - radiological progression group

* - 10/29 total surgical resection, ${ }^{\#}$-1/11 glioma transformed into glioblastoma and 10/11 gliomas transformed into anaplastic astrocytoma 
Table II. Parameters of PWI, DWI, ${ }^{1} \mathrm{H}-\mathrm{MRS}$ obtained in the contrast enhancement area and non-contrast enhancement area of the tumour, on the initial MR, in the stable group and the anaplastic transformation group

\begin{tabular}{|lccccccc|}
\hline \multirow{2}{*}{ Parameters } & \multicolumn{3}{c}{$\mathrm{ST}^{*}(n=10 / 29)$} & \multicolumn{3}{c|}{$\mathrm{AT}^{* *}(n=5 / 11)$} \\
\cline { 2 - 8 } & $\mathrm{CE}$ & Non-CE & $p$ & $\mathrm{CE}$ & Non-CE & $p$ \\
\hline Max nrCBV & $2.67(1.60-4.24)$ & $1.44(0.71-3.16)$ & 0.005 & $3.15(2.29-4.64)$ & $1.47(1.03-2.85)$ & 0.079 \\
\hline Mean nrCBV & $1.92(1.39-3.67)$ & $1.14(0.63-2.69)$ & 0.050 & $2.00(1.68-3.19)$ & $1.10(0.77-1.78)$ & 0.043 \\
\hline Mean Cho/Cr & $1.60(1.34-2.60)$ & $1.44(1.16-1.80)$ & 0.007 & $1.85(1.45-2.60)$ & $1.59(1.19-2.20)$ & 0.043 \\
\hline Mean Cho/NAA & $1.09(0.55-3.81)$ & $0.87(0.49-1.80)$ & 0.007 & $1.73(1.42-2.26)$ & $1.01(0.80-1.79)$ & 0.043 \\
\hline Mean NAA/Cr & $1.36(0.69-2.84)$ & $1.65(1.00-2.73)$ & NS & $1.10(0.82-1.51)$ & $1.41(0.89-2.79)$ & 0.043 \\
\hline Min ADC\# & $1.24(1.04-1.78)$ & $1.32(0.99-1.71)$ & NS & $0.98(0.70-1.14)$ & $1.29(0.81-1.43)$ & 0.043 \\
\hline Mean ADC\# & $1.31(1.13-1.86)$ & $1.46(1.13-1.79)$ & NS & $1.20(0.88-1.27)$ & $1.38(1.04-1.48)$ & 0.043 \\
\hline
\end{tabular}

ST - stable group, AT - anaplastic transformation group, CE - contrast enhancement area of the tumour, Non-CE - non-contrast enhancement area of the tumour

${ }^{\#} A D C$ values ${ }^{*} 10^{-3} \mathrm{~mm}^{2} / \mathrm{s}$, ${ }^{*}$ On the initial MR in the ST group 10/29 (34\%) tumours had CE area, ${ }^{* *}$ On the initial MR in the AT group, 5/11 (45\%) tumours had $C E$ area. The increase in max $n r C B V>1.75$ preceded contrast enhancement up to 5-8 months in those tumours which did not enhance on the initial MR

Table III. Statistically significant differences between the stable group and the anaplastic transformation group on the initial MR, follow-up MR and final MR

\begin{tabular}{|c|c|c|c|c|c|c|}
\hline \multicolumn{7}{|c|}{ Initial MR } \\
\hline & $\max n r C B V$ & mean nrCBV & & & & \\
\hline AT & $4.21 \pm 0.67$ & $2.48 \pm 0.69$ & & & & \\
\hline ST & $2.37 \pm 0.69$ & $1.47 \pm 0.49$ & & & & \\
\hline$p$ & 0.001 & 0.001 & & & & \\
\hline \multicolumn{7}{|c|}{ Follow-up MR } \\
\hline & $\max$ nrCBV & mean nrCBV & $\min A D C$ & mean ADC & & \\
\hline AT & $4.39 \pm 1.09$ & $2.56 \pm 0.62$ & $0.71(0.59-1.02)$ & $1.00(0.70-1.21)$ & & \\
\hline ST & $1.75 \pm 0.81$ & $1.13 \pm 0.40$ & $1.05(0.79-1.59)$ & $1.22(1.1-1.71)$ & & \\
\hline$p$ & 0.001 & 0.001 & 0.001 & 0.006 & & \\
\hline \multicolumn{7}{|c|}{ Final MR } \\
\hline & $\max$ nrCBV & mean nrCBV & $\min A D C$ & mean ADC & mean $\mathrm{Cho} / \mathrm{Cr}$ & mean Cho/NAA \\
\hline AT & $4.42 \pm 1.83$ & $2.93 \pm 1.31$ & $0.88(0.75-1.31)$ & $1.08(0.87-1.44)$ & $1.78(1.34-3.17)$ & $1.49(0.78-2.09)$ \\
\hline ST & $1.68 \pm 0.81$ & $1.01 \pm 0.44$ & $1.09(0.93-1.35)$ & $1.22(1.09-1.73)$ & $1.30(1.00-2.15)$ & $0.84(0.60-1.94)$ \\
\hline$p$ & 0.001 & 0.001 & 0.020 & 0.049 & 0.030 & 0.030 \\
\hline
\end{tabular}

AT - anaplastic transformation group, ST - stable group. In the ST group, max nrCBV and mean nrCBV values on the follow-up MR and final MR were significantly statistically lower compared to the results of the initial MR. Max nrCBV (2.37 \pm 0.69 vs. $1.75 \pm 0.81$ vs. $1.68 \pm 0.81 ; p=0.042, p=0.023)$. Mean nrCBV $(1.47 \pm 0.49$ vs. $1.13 \pm 0.4$ vs. $1.01 \pm 0.44 ; p=0.030, p=0.004)$.

In the AT group, on the follow-up MR, a statistically significant decrease in min $A D C$ and mean $A D C$ values in the remaining tumour area was observed compared to the initial MR examination. Min ADC (1.05 [0.71-1.36] vs. 0.71 [0.59-1.02]; $p=0.030)$. Mean ADC (1.29 [0.98-1.50] vs. 1.00 [0.70-1.21]; $p=0.030)$. Additionally, in the AT group an increase was observed in the value of Cho on the final MR compared to the initial MR examination. Cho/Cr (1.68 [1.10-2.19] vs. 1.78 [1.34-3.17]), Cho/NAA (1.32 [0.59-3.17] vs. $1.49[0.78-2.09])$

significantly lower compared to the non-enhancing area of the tumour (Table II).

Also in the ST group on the initial MR examination in the area of contrast enhancement, max nrCBV, mean nrCBV, mean Cho/Cr and mean Cho/NAA were significantly higher compared to the non-enhancing area of the tumour (Table II).
Compared to the ST group, tumours that underwent anaplastic transformation had statistically significantly higher values of mean nrCBV and max nrCBV on the initial MR examination, the follow-up and final MR examinations (Figs. 2 and 3, Table III). However, statistically significant differences between the groups in ADC values were observed on the fol- 


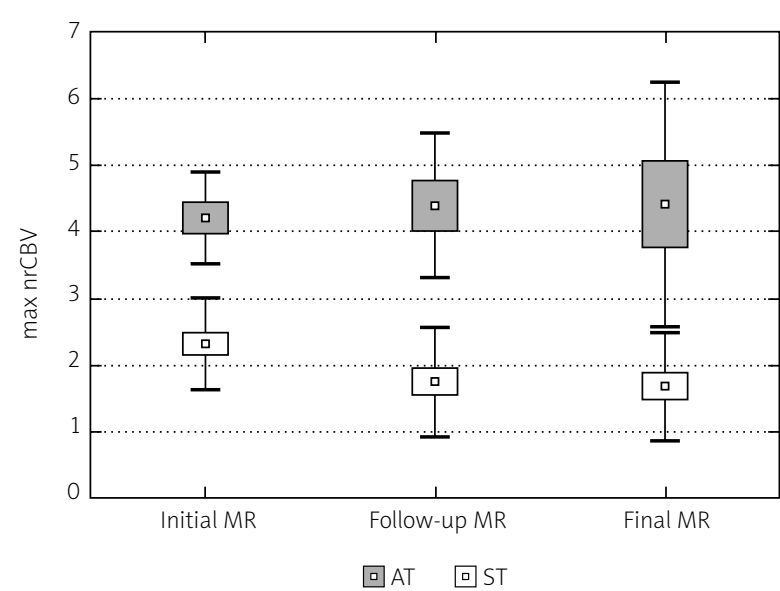

Fig. 2. Values of max nrCBV on the initial $M R$, follow-up MR and the final MR examinations in the stable group and the anaplastic transformation group.

low-up and final MR whereas mean $\mathrm{Cho/Cr}$ and mean Cho/NAA were observed as late as on the final MR examination (Figs. 4A-H and 5A-J, Table III).

\section{Discussion}

Magnetic resonance imaging is a non-invasive method of evaluating certain features of the tumour assessed by pathological examination, for instance tissue microvascular density (MVD), cellularity, the presence of necrosis [4,5,12,15,32,34,38,50]. Malignancy is characterised by vascular proliferation within the tumour (neoangiogenesis) $[6,22,30,39,47]$. According to the "angiogenic switch" theory, the essence of LGG anaplastic transformation is vascular proliferation in a previously avascular tumour $[22,47]$. Vessels formed by neoangiogenesis differ in their structure from normal vasculature (tortuosity, lack of maturity and increased permeability). On standard MR imaging, neoangiogenesis manifests as the area of contrast enhancement. Contrast enhancement as a marker of anaplastic transformation appears late and its appearance proves that anaplastic transformation has already occurred. However, PWI gives access to information on the capillary microcirculation of tissues and reflects tissue MVD by measuring rCBV, prior to the occurrence of contrast enhancement [5,35]. Danchaivijitr et al. confirmed that in the group of gliomas which underwent anaplastic transformation, continuous rCBV growth (within 1218 months) preceded the occurrence of an abnormal

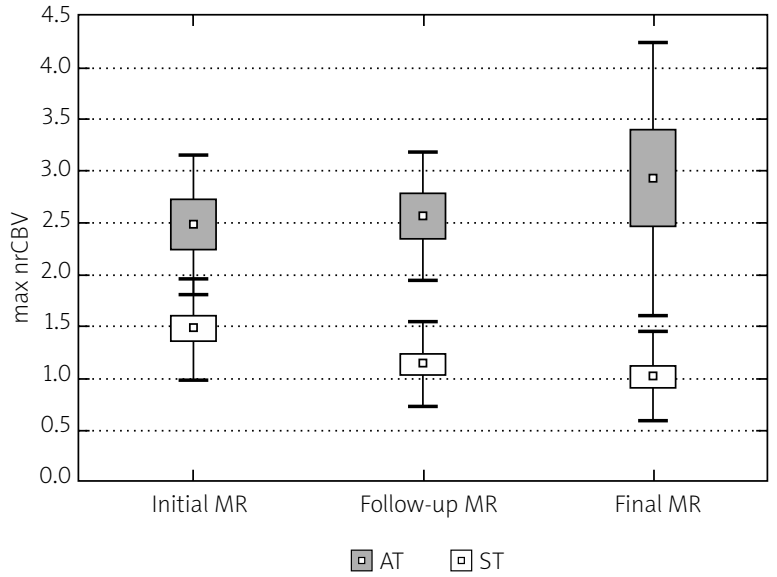

Fig. 3. Values of mean nrCBV on the initial $M R$, follow-up $M R$ and the final MR examinations in the stable group and the anaplastic transformation group.

area of contrast enhancement in the tumour [13]. Law et al. suggested the cut-off value for the normalised to the normal appearing white matter rCBV $>1.75$ on MR examination prior to surgery as the feature differentiating rapidly progressive LGGs (median 8 vs. 154 months) [24,26]. The similar correlation was confirmed by Caseiras et al. using the same cutoff value for rCBV - "the median time to progression among subjects with rCBV > 1.75 was 365 days, while there was $95 \%$ confidence that the median time to progression was at least 889 days among subjects with $\mathrm{rCBV}<1.75$ " [7]. Referring to the findings of Law et al., Al-Okaili et al. used the same cut-off value for rCBV characterising the area of the increased perfusion in the study on differential diagnosis of intra-axial brain masses. Gliomas with rCBV $>1.75$ corresponded to HGGs with sensitivity of $88 \%$, specificity of $100 \%$, PPV of $100 \%$, NPV of $67 \%$, compared to LGGs [1]. Although the normalised $\mathrm{rCBV}=$ 1.75 is the most frequently accepted cut-off value differentiating LGGs from HGGs as well as LGGs with short and long transformation time, other clearly distinct values are also reported. In the study by Arvinda et al., the cut-off point for nrCBV mostly differentiating LGGs from HGGs was 2.93, whereas Morita et al. obtained nrCBV $=0.94$ [2,33]. In our material in the AT group, the increase in max nrCBV > 1.75 preceded contrast enhancement in the same tumour area within 5-8 months in 6/11 (45\%) patients. In the remaining $5 / 11$ (55\%) patients, max nrCBV > 1.75 was found in the area of contrast enhancement from 

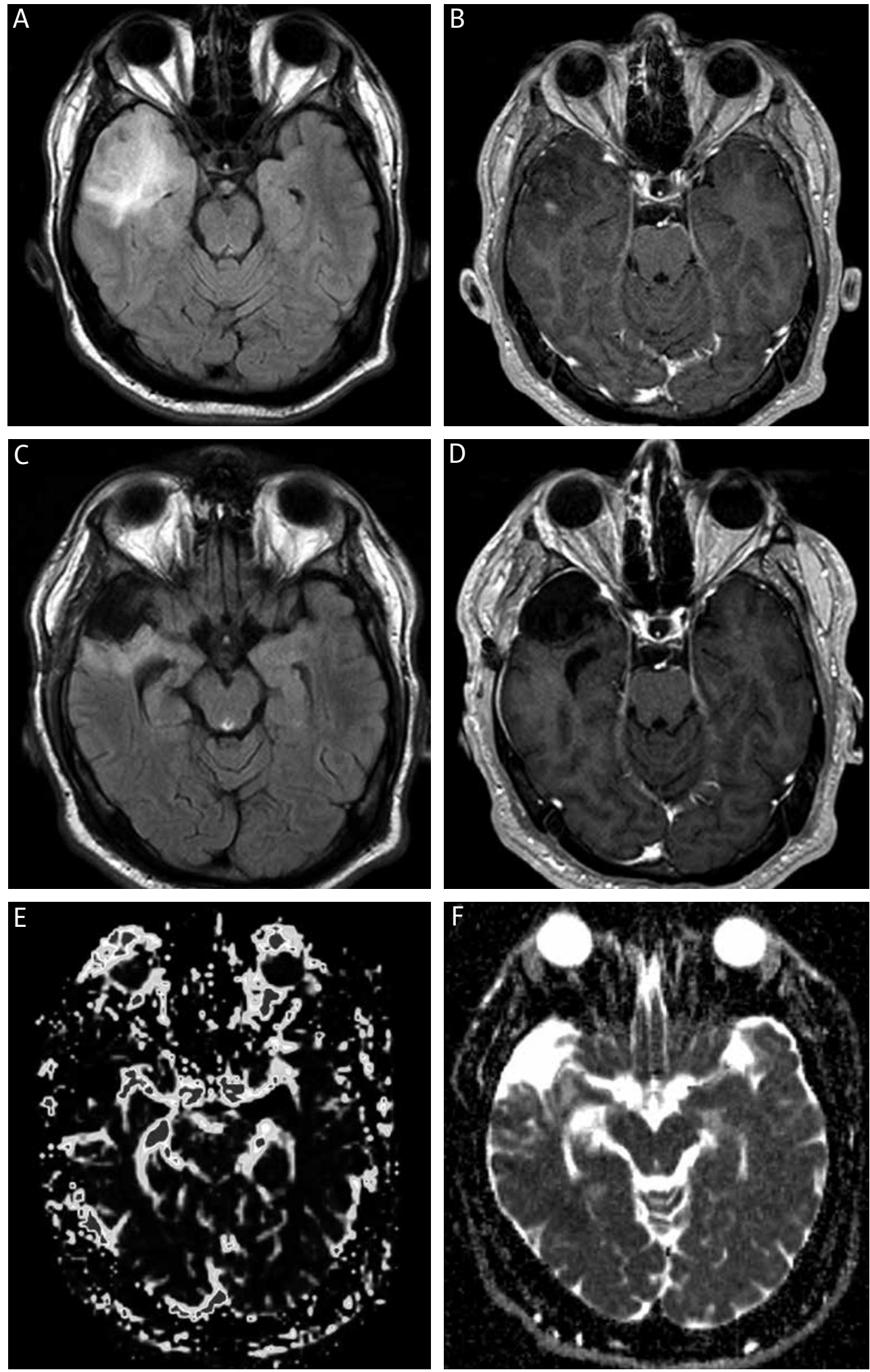

Fig. 4. A-F) Fibrillary astrocytoma WHO II out of the stable group, the initial MR: FLAIR (A) and T1-weighted image after intravenous contrast agent application (B), the final MR: FLAIR (C) and T1-weighted image after intravenous contrast agent application (D), PWI - rCBV map (E), DWI - ADC map (F). 

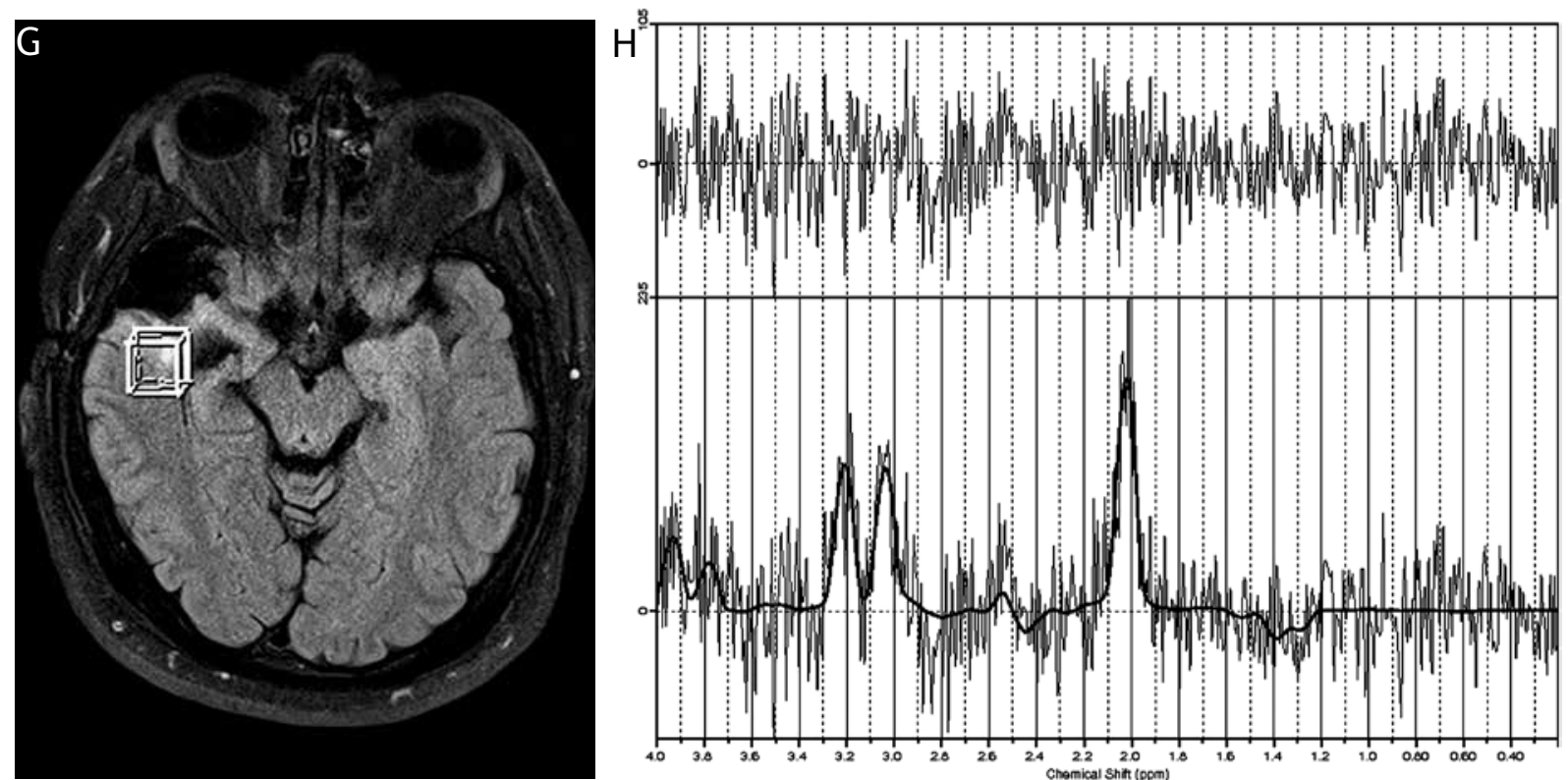

Fig. 4. G-H) Fibrillary astrocytoma WHO II out of the stable group, the initial MR: ${ }^{1} \mathrm{H}-\mathrm{MRS}$.

the very beginning. Moreover, max nrCBV and mean $\mathrm{nrCBV}$ in AT group were significantly higher on the initial MR, follow-up MR and the final MR examinations compared to the ST group. In the ST group max $n r C B V$ and mean nrCBV were significantly lower on the follow-up MR and the final MR examinations compared to the initial MR examination, which may have been due to resection of contrast enhancement area characterised by significantly increased nrCBV in relation to the remaining tumour area.

In our material, the extent of tumour resection in the ST group was significantly higher compared to the AT group. The extent of tumour resection significantly influenced progression-free survival (PFS) and overall survival (OS) in LGG patients, which is consistent with the reports of other researchers $[19,31,43]$. Our observations confirm that next to the extent of resection, excision of contrast enhancement area is essential for further course of disease. In our material, none of the patients from the AT group had contrast enhancement area resected whereas in the ST group contrast enhancement area was resected in all patients. In both groups in the area of contrast enhancement nrCBV and $\mathrm{Cho} / \mathrm{Cr}$ and $\mathrm{Cho} / \mathrm{NAA}$ ratios were significantly higher compared to the non-enhanced tumour area, which indicates higher potential malignancy of this part of tumour. Additionally, in the AT group in the area of contrast enhancement
ADC was significantly lower compared to the outside of this area. Chaichan et al. confirmed that in patients with LGGs enhanced after contrast agent administration, the 5-year OS, PFS and malignant transformation-free survival were shorter compared to patients with non-enhancing LGGs (70 vs. $85 \%$ [ $p=0.002$ ], 32 vs. $49 \%$ [ $p=0.008]$ and 74 vs. $90 \%$ $[p=0.002]$, respectively). Moreover, the only factors that were consistently shown to be associated with tumour recurrence or malignant degeneration are preoperative contrast enhancement, tumour size, and subtotal resection. Additionally, in the multifactorial analysis, Chaichan et al. confirmed that LGG contrast enhancement is an independent factor determining decreased survival $(p=0.006)$, increased recurrence $(p=0.04)$ and the trend towards a higher incidence of malignant degeneration $(p=0.15)$. However, Chaichan et al. did not analyse parameters of PWI, DWI, proton MR spectroscopy ( $\left.{ }^{1} \mathrm{H}-\mathrm{MRS}\right)[9,10]$. The criteria for Response Assessment in Neuro-Oncology (RANO) regarding the assessment of response to treatment of non-enhancing LGGs indicate that the area of contrast enhancement in the tumour corresponds to malignant transformation although it is a non-specific feature [45], and parameters of PWI, DWI, ${ }^{1} \mathrm{H}-\mathrm{MRS}, \mathrm{PET}$ though mentioned in the model protocol are not included in the treatment-response assessment. According to the authors of the RANO 

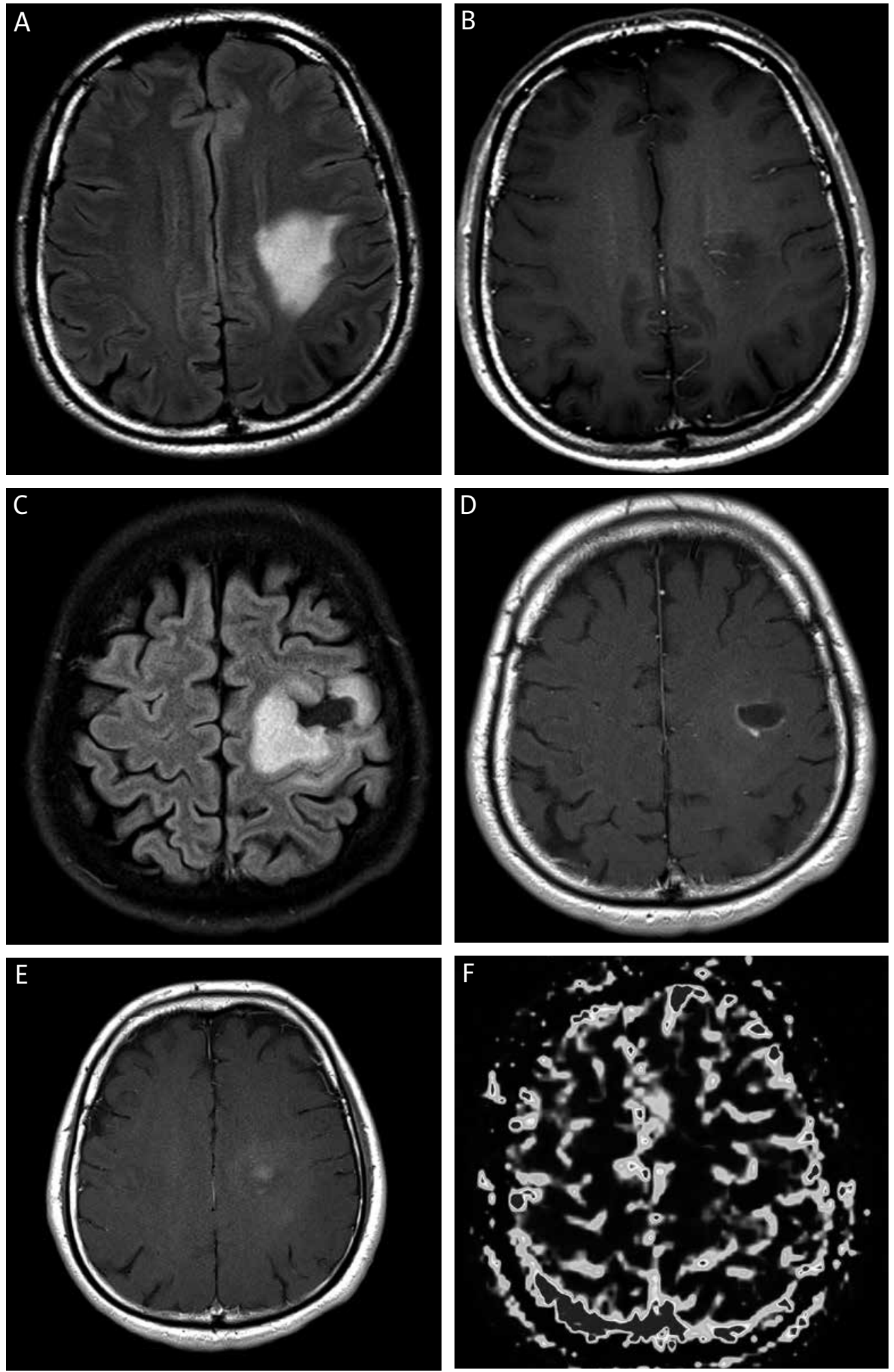

Fig. 5. A-F) Fibrillary astrocytoma WHO II out of the anaplastic transformation group, the initial MR: FLAIR (A) and T1-weighted image after intravenous contrast agent application (B), the final MR: FLAIR (C) and T1-weighted image after intravenous contrast agent application (D, E), PWI - rCBV map (F, G). 

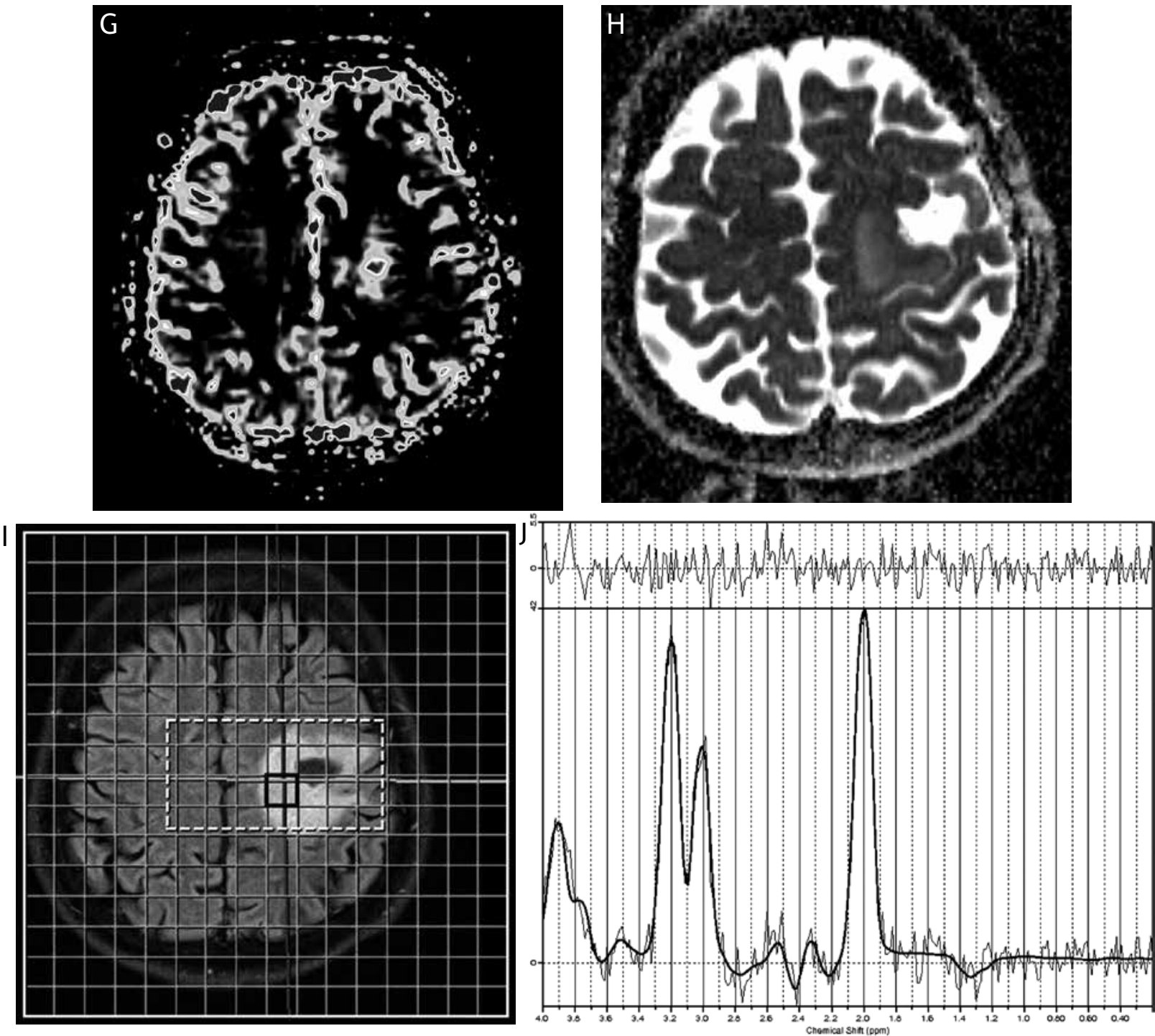

Fig. 5. G-J) Fibrillary astrocytoma WHO II out of the anaplastic transformation group, the initial MR: PWI rCBV map (F, G), DWI - ADC map (H), ${ }^{1} \mathrm{H}-\mathrm{MRS}(\mathrm{I}, \mathrm{J})$.

criteria, the percentage of gliomas WHO II enhancing after contrast agent administration is reported to be at the level of $10 \%$ and mainly in the oligodendroglioma subgroup. The post-treatment occurrence of a new area of contrast enhancement in gliomas WHO II is first interpreted as malignant transformation [45]. According to RANO, "for progression, two types of MRI progression have to be distinguished and reported separately: the development of or an increase in enhancement suggestive of malignant transformation, and linear progression of a still non-enhancing lesion".

In our study, astrocytomas and oligoastrocytomas occurred in both groups (ST and AT) whereas oligo- dendrogliomas exclusively in the ST group. A gemistocytic component was found more frequently in the AT group (36\% of tumours) compared to the ST group ( $21 \%$ of tumours).

In the multifactorial analysis regarding early prognostic factors related to progression and malignant transformation of LGGs, Joung et al. confirmed that apart from total tumour resection, a gemistocytic component in LGGs was related to shorter PFS and independently influenced the occurrence of malignant transformation [19]. Additionally, in trials regarding LGG treatment with radiotherapy, the median survival of $>5$ years was reported for patients with oligodendroglioma compared to other 
gliomas WHO II for which the median survival was $<5$ years $[20,42,44]$.

There are no explicit reports on the ADC cutoff value differentiating gliomas depending on the tumour grade $[2,8,41,46]$. However, it is generally accepted that the higher the tumour grade, the lower ADC values [46]. Server et al. and Arvinda et al. suggested the minimum $A D C=1.07 \times 10^{-3} \mathrm{~mm}^{2} / \mathrm{s}$ and the mean $A D C=0.985 \times 10^{-3} \mathrm{~mm}^{2} / \mathrm{s}$ as the $A D C$ cut-off values differentiating HGGs from LGGs $[2,41]$. However, Catalaa et al. noted that HGGs and LGGs did not differ in $A D C$ values [8]. In our material, min $A D C$ and mean ADC did not differ significantly between AT and ST groups on the initial MR examination. However, min ADC and mean ADC were significantly lower in the AT group on the follow-up MR and the final MR examinations compared to the ST group. In the AT group the follow-up MR revealed a statistically significant decrease in min ADC and mean $A D C$ values in the remaining tumour area compared to the initial MR examination. It may be related to the increased component of post-treatment ischaemic changes or gliosis. It cannot be clearly determined which process or component mainly contributed to the decrease in the ADC value.

The cut-off value for $\mathrm{Cho} / \mathrm{Cr}$ and Cho/NAA reported by various researchers differentiating LGGs from HGGs is wide (1.35 to 2.04 for $\mathrm{Cho} / \mathrm{Cr}$ and 1.60 to 2.49 for Cho/NAA) $[14,23,29,41,49]$. Hlaihel et al. confirmed that in the group of oligodendrogliomas WHO II which underwent anaplastic transformation, mean $\mathrm{Cho} / \mathrm{Cr}$ ratio above 2.4 was the earliest marker of the transformation with a mean delay of 15.4 months. Whereas normalised rCBV elevation $>2$ occurred an average of 8 months before the malignant transformation. Authors suggested that "the increase in cell membrane production and cell density, as attested by the elevation of the choline/creatine ratio, may generate an ischaemic process that initiates vascular proliferation in the infiltrative tissue" [18]. In our study, in the AT group mean $\mathrm{Cho} / \mathrm{Cr}$ and mean Cho/NAA were significantly higher as late as on the final MR examination compared to the ST group. The increase in Cho over time was observed in the AT group, i.e. mean $\mathrm{Cho} / \mathrm{Cr}$ and mean Cho/NAA were higher on the final $M R$ examination compared to the initial $M R$ examination. Our analysis comprised of ${ }^{1} \mathrm{H}-\mathrm{MRS}$ with a long TE: 135-288 ms, whereas Hlaihel et al. analysed mainly single-voxel short TE: $32 \mathrm{~ms}^{1} \mathrm{H}$-MRS. Moreover, in our material there were different gliomas WHO ॥ (fibrillary astrocytoma, gemistocytic astrocytoma, oligodendroglioma, oligoastrocytoma), whereas Hlaihel et al. analysed only oligodendrogliomas. We had longer follow-up and more gliomas which underwent anaplastic transformation.

\section{Conclusions}

Multiparametric MR examination allows the detection of LGGs with high probability of rapid anaplastic transformation and the detection of transformation prior to the occurrence of contrast enhancement. The value of nrCBV is the most useful in the diagnosis of anaplastic transformation. The resection of contrast enhancement area of the tumour significantly increases time to anaplastic transformation of LGGs.

\section{Acknowledgements}

The authors would like to thank Arkadiusz Badziński for translating the article.

\section{Disclosure}

This study was supported by the Ministry of Science and Higher Education grant no. NN 403278933 which was approved by the local research ethics committee.

Authors report no conflict of interest.

\section{References}

1. Al-Okaili RN, Krejza J, Woo JH, Wolf RL, O’Rourke DM, Judy KD, Poptani H, Melhem ER. Intraaxial brain masses: MR imaging-based diagnostic strategy - initial experience. Radiology 2007; 243: 539-550.

2. Arvinda HR, Kesavadas C, Sarma PS, Thomas B, Radhakrishnan VV, Gupta AK, Kapilamoorthy TR, Nair S. Glioma grading: sensitivity, specificity, positive and negative predictive values of diffusion and perfusion imaging. I Neurooncol 2009; 94: 87-96.

3. Brady LW, Heilmann HP, Molls M, Neider C. Radiation Oncology an evidence based approach. Springer-Verlag, Berlin, Heidelberg 2008, pp. 483-499.

4. Bobek-Billewicz B, Hebla A, Stasik-Pres G, Majchrzak K, Żmuda E, Trojanowska A. Measurement of glycine in a brain and brain tumors by means of 1H MRS. Folia Neuropathol 2010; 48: 190-199.

5. Bobek-Billewicz B, Stasik-Pres G, Majchrzak H, Zarudzki L. Differentiation between brain tumor recurrence and radiation injury using perfusion, diffusion-weighted imaging and MR spectroscopy. Folia Neuropathol 2010; 48: 81-92.

6. Carmeliet P, Jain RK. Angiogenesis in cancer and other diseases. Nature 2000; 407: 249-257.

7. Caseiras GB, Chheang S, Babb J, Rees JH, Pecerrelli N, Tozer DJ, Benton C, Zigzag D, Johnson G, Waldman AD, Jäger HR, Law M. 
Relative cerebral blood volume measurements of low-grade gliomas predict patient outcome in a multi-institution setting. Eur J Radiol 2010; 73: 215-220.

8. Catalaa I, Henry R, Dillon WP, Graves EE, McKnight TR, Lu Y, Vigneron DB, Nelson SJ. Perfusion, diffusion and spectroscopy values in newly diagnosed cerebral gliomas. NMR Biomed 2006; 19: 463-475.

9. Chaichana KL, McGirt MJ, Niranjan A, Olivi A, Burger PC, Quinones-Hinojosa A. Prognostic significance of contrast-enhancing low-grade gliomas in adults and a review of the literature. Neurol Res 2009; 31: 931-939.

10. Chaichana KL, McGirt MJ, Laterra J, Olivi A, Quiñones-Hinojosa A. Recurrence and malignant degeneration after resection of adult hemispheric low-grade gliomas. J Neurosurg 2010; 112: 10-17.

11. Crocetti E, Trama A, Stiller C, Caldarella A, Soffietti R, Jaal J, Weber DC, Ricardi U, Slowinski J, Brandes A; RARECARE working group. Epidemiology of glial and non-glial brain tumours in Europe. Eur J Cancer 2012; 48: 1532-1542.

12. Czernicki T, Szeszkowski W, Marchel A, Gołebiowski M. Spectral changes in postoperative MRS in high-grade gliomas and their effect on patient prognosis. Folia Neuropathol 2009; 47: 43-49.

13. Danchaivijitr N, Waldman AD, Tozer DJ, Benton CE, Brasil Caseiras G, Tofts PS, Rees JH, Jäger HR. Low-grade gliomas: do changes in rCBV measurements at longitudinal perfusion-weighted MR imaging predict malignant transformation? Radiology 2008; 247: 170-178.

14. Delorme S, Weber MA. Applications of MRS in the evaluation of focal malignant brain lesions. Cancer Imaging 2006; 6: 95-99.

15. Dhermain FG, Hau P, Lanfermann H, Jacobs AH, van den Bent MJ. Advanced MRI and PET imaging for assessment of treatment response in patients with gliomas. Lancet Neurol 2010; 9: 906 920.

16. Didkowska J, Wojciechowska U, Zatonski W. Nowotwory złośliwe w Polsce w 2009 roku. Centrum Onkologii - Instytut, Warszawa 2011.

17. Henson JW, Ulmer S, Harris GJ. Brain tumor imaging in clinical trials. AJNR Am J Neuroradiol 2008; 29: 419-424.

18. Hlaihel C, Guilloton L, Guyotat J, Streichenberger N, Honnorat J, Otton F. Predictive value of multimodality MRI using conventional, perfusion, and spectroscopy MR in anaplastic transformation of low-grade oligodendrogliomas. J Neurooncol 2010; 97: 73-80.

19. Jung TY, Jung S, Moon JH, Kim IY, Moon KS, Jang WY. Early prognostic factors related to progression and malignant transformation of low-grade gliomas. Clin Neurol Neurosurg 2011; 113 752-757.

20. Karim AB, Maat B, Hatlevoll R, Menten J, Rutten EH, Thomas DG, Mascarenhas F, Horiot JC, Parvinen LM, van Reijn M, Jager JJ, Fabrini MG, van Alphen AM, Hamers HP, Gaspar L, Noordman E, Pierart M, van Glabbeke M. A randomized trial on dose-response in radiation therapy of low-grade cerebral glioma: European Organization for Research and Treatment of Cancer (EORTC) Study 22844. Int J Radiat Oncol Biol Phys 1996; 36: 549-556.

21. Krzakowski M, Fijuth M, Herman K, Jarosz J, Jassem J, Kawecki A, Kornafel J, Krzemieniecki K, Litwiniuk M, Potemski P, Rubach M, Rutkowski P, Stelmach A. Onkologia w praktyce klinicznej. Zalece- nia postępowania diagnostyczno-terapeutycznego w nowotworach złośliwych. Tom I. Via Medica, Gdańsk 2011, pp. 33-67.

22. Lacerda S, Law M. Magnetic resonance perfusion and permeability imaging in brain tumors. Neuroimaging Clin N Am 2009; 19: 527-557.

23. Law M, Yang S, Wang H, Babb JS, Johnson G, Cha S, Knopp EA, Zagzag D. Glioma grading: sensitivity, specificity, and predictive values of perfusion MR imaging and proton MR spectroscopic imaging compared with conventional MR imaging. AJNR Am J Neuroradiol 2003; 24: 1989-1998.

24. Law M, Oh S, Babb JS, Wang E, Inglese M, Zagzag D, Knopp EA, Johnson G. Low-grade gliomas: dynamic susceptibility-weighted contrast-enhanced perfusion MR imaging-prediction of patient clinical response. Radiology 2006; 238: 658-667.

25. Law M, Yang S, Babb JS, Knopp EA, Golfinos JG, Zagzag D, Johnson $\mathrm{G}$. Comparison of cerebral blood volume and vascular permeability from dynamic susceptibility contrast-enhanced perfusion MR imaging with glioma grade. AJNR Am J Neuroradiol 2004; 25: 746-755.

26. Law M, Young RJ, Babb JS, Peccerelli N, Chheang S, Gruber ML, Miller DC, Golfinos JG, Zagzag D, Johnson G. Gliomas: predicting time to progression or survival with cerebral blood volume measurements at dynamic susceptibility-weighted contrast-enhanced perfusion MR imaging. Radiology 2008; 247: 490-498.

27. Lesniak MS, Klem JM, Weingart J, Carson BS. Surgical outcome following resection of contrast-enhanced pediatric brainstem gliomas. Pediatr Neurosurg 2003; 39: 314-322.

28. Liu X, Tian W, Kolar B, Yeaney GA, Qiu X, Johnson MD, Ekholm S. $M R$ diffusion tensor and perfusionweighted imaging in preoperative grading of supratentorialnonenhancing gliomas. Neuro Oncol 2011; 13: 447-455.

29. Liu ZL, Zhou Q, Zeng QS, Li CF, Zhang K. Noninvasive evaluation of cerebral glioma grade by using diffusion-weighted imaging-guided single-voxel proton magnetic resonance spectroscopy. J Int Med Res 2012; 40: 76-84.

30. Louis DN, Ohgaki H, Wiestler OD, Cavenee WK. 4th Edition WHO Classification of Tumours of the Central Nervous System. International Agency for Research on Cancer, Lyon 2007.

31. Majchrzak K, Kaspera W, Bobek-Billewicz B, Hebda A, StasikPres G, Majchrzak H, Ladziński P. The assessment of prognostic factors in surgical treatment of low-grade gliomas: A prospective study. Clin Neurol Neurosurg 2012; 114: 1135-1144.

32. Matulewicz L, Sokół M, Wydmański J, Hawrylewicz L. Could lipid $\mathrm{CH} 2 / \mathrm{CH} 3$ analysis by in vivo $1 \mathrm{H}$ MRS help in differentiation of tumor recurrence and post-radiation effects? Folia Neuropathol 2006; 44: 116-124.

33. Morita N, Wang S, Chawla S, Poptani H, Melhem ER. Dynamic susceptibility contrast perfusion weighted imaging in grading of nonenhancing astrocytomas. J Magn Reson Imaging 2010; 32: 803-808

34. Provenzale JM. Imaging of angiogenesis: clinical techniques and novel imaging methods. AJR Am J Roentgenol 2007; 188: 11-23.

35. Quant EC, Wen PY. Response assessment in neuro-oncology. Curr Oncol Rep 2011; 13: 50-56.

36. Ręcławowicz D, Stempniewicz M, Biernat W, Limon J, Stoniewski P. Loss of genetic material within $1 p$ and $19 q$ chromosom- 
al arms in low grade gliomas of central nervous system. Folia Neuropathol 2013; 51: 26-32.

37. Romanowski CAJ, Hoggard N, Jellinek DA, Levy D, Wharton SB, Kotsarini C, Batty R, Wilkinson ID. Low grade gliomas. Can we predict tumour behaviour from imaging features? NRJ 2008; 21 41-66.

38. Rowley HA, Grant P, Roberts T. Diffusion MR imaging. Theory and applications. Neuroimaging Clin N Am 1999; 9: 343-361.

39. Russell SM, Elliott R, Forshaw D, Golfinos JG, Nelson PK, Kelly PJ. Glioma vascularity correlates with reduced patient survival and increased malignancy. Surg Neurol 2009; 72: 242-247.

40. Schomas DA, Laack NN, Rao RD, Meyer FB, Shaw EG, O'Neill BP, Giannini C, Brown PD. Intracranial low-grade gliomas in adults: 30-year experience with long-term follow-up at Mayo Clinic Neuro Oncol 2009; 11: 437-445.

41. Server A, Kulle B, Gadmar ØB, Josefsen R, Kumar T, Nakstad PH. Measurements of diagnostic examination performance using quantitative apparent diffusion coefficient and proton MR spectroscopic imaging in the preoperative evaluation of tumor grade in cerebral gliomas. Eur J Radiol 2011; 80: 462-470.

42. Shaw E, Arusell R, Scheithauer B, O'Fallon J, O'Neill B, Dinapoli R, Nelson D, Earle J, Jones C, Cascino T, Nichols D, Ivnik R, Hellman R, Curran W, Abrams R. Prospective randomized trial of low- versus high-dose radiation therapy in adults with supratentorial low-grade glioma: initial report of a North Central Cancer Treatment Group/Radiation Therapy Oncology Group/Eastern Cooperative Oncology Group study. J Clin Oncol 2002; 20: 2267-2276.

43. Smith JS, Chang EF, Lamborn KR, Chang SM, Prados MD, Cha S, Tihan T, Vandenberg S, McDermott MW, Berger MS. Role of extent of resection in the long-term outcome of low-grade hemispheric gliomas. J Clin Oncol 2008; 26: 1338-1145.

44. van den Bent MJ, Afra D, de Witte O, Ben Hassel M, Schraub S, Hoang-Xuan K, Malmström PO, Collette L, Piérart M, Mirimanoff R, Karim AB; EORTC Radiotherapy and Brain Tumor Groups and the UK Medical Research Council. Long-term efficacy of early versus delayed radiotherapy for low-grade astrocytoma and oligodendroglioma in adults: the EORTC 22845 randomised trial. Lancet 2005; 366: 985-990. Erratum in: Lancet 2006; 367: 1818.

45. van den Bent MJ, Wefel JS, Schiff D, Taphoorn MJ, Jaeckle K, Junck L, Armstrong T, Choucair A, Waldman AD, Gorlia T, Chamberlain M, Baumert BG,Vogelbaum MA, Macdonald DR, Reardon DA, Wen PY, Chang SM, Jacobs AH. Response assessment in neuro-oncology (a report of the RANO group): assessment of outcome in trials of diffuse low-grade gliomas. Lancet Oncol 2011; 12: 583-593.

46. Weber MA, Henze M, Tüttenberg J, Stieltjes B, Meissner M, Zimmer F, Burkholder I, Kroll A, Combs SE, Vogt-Schaden M, Giesel FL, Zoubaa S, Haberkorn U, Kauczor HU, Essig M. Biopsy targeting gliomas: do functional imaging techniques identify similar target areas? Invest Radiol 2010; 45: 755-768.

47. Verheul HM, Voest EE, Schlingemann RO. Are tumours angiogenesis-dependent? J Pathol 2004; 202: 5-13.

48. Wessels PH, Weber WE, Raven G, Ramaekers FC, Hopman AH, Twijnstra A. Supratentorial grade II astrocytoma: biological features and clinical course. Lancet Neurol 2003; 2: 395-403.
49. Zeng Q, Liu H, Zhang K, Li C, Zhou G. Noninvasive evaluation of cerebral glioma grade by using multivoxel 3D proton MR spectroscopy. Magn Reson Imaging 2011; 29: 25-31.

50. Zhang W, Zhao J, Guo D, Zhong W, Shu J, Luo Y. Application of susceptibility weighted imaging in revealing intratumoral blood products and grading gliomas. J Radiol 2010; 91: 485-490. 